\title{
Sharp peaks in the momentum distribution of bosons in optical lattices in the normal state
}

\author{
YASUYUKI KATO ${ }^{1}$, QI ZHOU², NAOKI KAWASHIMA ${ }^{1}$ AND NANDINI TRIVEDI2* \\ ${ }^{1}$ Institute for Solid State Physics, University of Tokyo, 5-1-5 Kashiwa-no-ha, Kashiwa, Chiba 277-8581, Japan \\ ${ }^{2}$ Department of Physics, The Ohio State University, Columbus, Ohio 43210, USA \\ *e-mail: trivedi.15@osu.edu
}

Cold atoms in optical lattices provide a unique laboratory for investigating quantum phase transitions between strongly correlated superfluid and Mott insulator phases ${ }^{1,2}$. One of the major bottlenecks in the analysis of experiments is a clear set of criteria for identifying the superfluid phase ${ }^{3}$. A 'sharp' interference pattern in time-of-flight experiments has been widely adopted as a signature of superfluidity ${ }^{4-8}$. Here, we show that sharp peaks are not a reliable diagnostic of superfluidity. Using large-scale quantum Monte Carlo simulations of the Bose-Hubbard model in three dimensions with up to $N=1.4 \times 10^{4}$ particles, we calculate the momentum distribution $n(k)$ as a function of temperature $T$ and $t / U$, the ratio of hopping to the onsite repulsion. We find that even above the transition temperature $T_{c}$ where both superfluid density and condensate fraction vanish, the interference pattern can nevertheless have sharp peaks riding over a broad background. We identify the true signature of the superfluid and give a deeper understanding of why such sharp peaks appear in the normal state.

Bosons in an optical lattice are well described by the single-band Bose-Hubbard model ${ }^{9,10}$ when the occupancy in higher bands can be neglected, given by,

$$
H=-\frac{t}{z} \sum_{\langle i, j\rangle}\left(b_{i}^{+} b_{j}+b_{j}^{+} b_{i}\right)+\frac{U}{2} \sum_{i} n_{i}\left(n_{i}-1\right)-\mu \sum_{i} n_{i},
$$

where $b_{i}\left(b_{i}^{+}\right)$is the boson destruction (creation) operator at a site $i, n_{i}=b_{i}^{+} b_{i}$ is the density operator and $z=6$ is the coordination number in three dimensions. Both $t$ and $U$ are calculated directly for a given lattice depth $V_{0}$, which can be tuned by the laser intensity ${ }^{10}$. At large $t / U$, the system is a normal Bose gas at high $T$ and undergoes a classical phase transition to a superfluid at the transition temperature $T_{\mathrm{c}}$. As $t / U$ decreases, $T_{\mathrm{c}}$ is suppressed and eventually becomes zero at $(t / U)_{c}$, the quantum critical point, as shown in Fig. 1a. As $t / U$ is reduced further, the system becomes a Mott insulator with a gap in the excitation spectrum. In the quantum critical region, the bosons are strongly interacting and highly incoherent as the degrees of freedom reorganize between well-defined collective modes in the superfluid to well-defined single-particle modes in the Mott insulator.

To identify the phases, we concentrate on the momentum distribution $n(\mathbf{k})$, a quantity of fundamental interest that encapsulates the strong correlations of the system. $n(\mathbf{k})$ is measured in time-of-flight experiments by releasing the atomic cloud from the optical lattice potential and the magnetic trap. Given the strong confinement energy released from each site in the optical lattice, the expansion is largely ballistic and the calculated $n(\mathbf{k})$ then represents the interference pattern ${ }^{11}$. Here, we ignore interaction effects in the expansion, which is a small perturbation on the final observed interference pattern. The final density distribution $\tilde{n}(\mathbf{r})$ in the interference pattern is ${ }^{12}$

$$
\tilde{n}(\mathbf{r})=\left(\frac{m}{\hbar \tau}\right)^{3}\left|W\left(\mathbf{k}=\frac{m \mathbf{r}}{\hbar \tau}\right)\right|^{2} n\left(\mathbf{k}=\frac{m \mathbf{r}}{\hbar \tau}\right),
$$

where $W(\mathbf{k})$ is the Fourier transform of the Wannier function in the lattice, $\tau$ is the expansion time and

$$
n(\mathbf{k})=\sum_{i, j}\left\langle b_{i}^{+} b_{j}\right\rangle \mathrm{e}^{i \mathbf{k} \cdot\left(\mathbf{r}_{i}-\mathbf{r}_{j}\right)}
$$

is the momentum distribution function of bosons before expansion and is the central quantity of interest. The final image detects the column-integrated momentum distribution $N_{\perp}\left(k_{x}, k_{y}\right)=\int \mathrm{d} k_{z}|W(\mathbf{k})|^{2} n(\mathbf{k})$. Typically, the interference pattern has two distinct types of behaviour: (1) a blob considered to be representative of the Mott insulating incoherent phase, and (2) a bimodal structure, with a broad background above which there are visibly sharp peaks located at the reciprocal lattice vectors. The peaks are well separated from each other with a width less than the reciprocal lattice vector. The presence of sharp peaks has been considered an unequivocal signature of superfluidity in the Bose system ${ }^{4,5}$.

The questions that arise are as follows. (1) Are sharp peaks in the interference pattern a reliable diagnostic of superfluidity? (2) What is the fate of the sharp peaks as the interactions between bosons are increased at a fixed temperature moving towards the quantum phase transition? (3) Furthermore, at the quantum critical point, what happens to the interference pattern as $T$ is reduced in a regime with no superfluidity?

To include the effect of strong interactions 'exactly', we use the world-line quantum Monte Carlo method ${ }^{13,14}$, applied to the hamiltonian in equation (1), based on the general idea of the directed-loop algorithm with modifications to improve efficiency near a critical point ${ }^{15}$. The accuracy of the method is tested in various ways by comparisons with exact diagonalization for small systems, and checks on the exponents with known results 
a

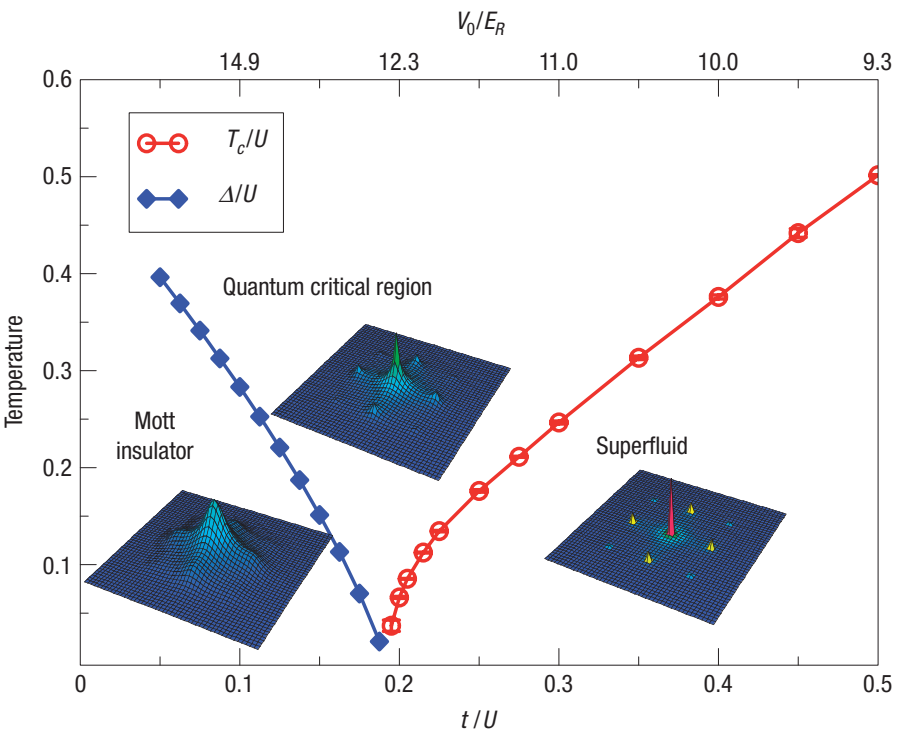

b

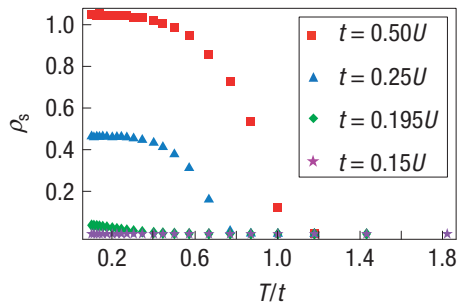

$\mathbf{G}$

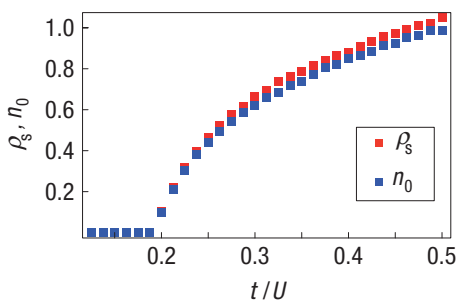

Figure 1 Phase diagram. a, The superfluid transition temperature $T_{\mathrm{c}}$ (red) and the single-particle energy gap $\Delta$ in the Mott insulator (blue) vanish at the quantum phase transition as a function of tuning $t / U$, the ratio of hopping to onsite repulsion. For $\mu / U=1 / 2,(t / U)_{\mathrm{c}}=0.192(2)$. The corresponding lattice depth values $V_{0}$ in units of the recoil energy $E_{\mathrm{R}}=h^{2} / 8 m d^{2}$ are indicated on the top axis. Here, $m$ is the mass of the rubidium atom and $d$ is the lattice constant. The typical interference patterns in the superfluid, Mott and critical regions are also shown. b. The superfluid density $\rho_{\mathrm{s}}$ in units of $1 / d^{3}$ as a function of $T$ for various $t / U$. c, The superfluid density $\rho_{\mathrm{s}}$ and the condensate density $n_{0}$ both in units of $1 / d^{3}$ as a function of $t / U$ at $T=0.1 t$.

for the three-dimensional XY model. To further test that the true equilibrium distribution is sampled on large systems, several independent runs with varying length are carried out, showing no systematic deviation, thereby ensuring that our numerical results are 'exact' except for statistical errors.

We calculate the correlations and excitations of a Bose-Hubbard model as a function of $t / U$ and temperature $T$ at fixed $\mu / U=0.5$. As shown in Fig. 1a, the Mott phase is identified by a finite single-particle gap $\Delta$ obtained from the imaginary time decay of Green's function. The superfluid density $\rho_{\mathrm{s}}=T\left\langle W^{2}\right\rangle /(L t)$ is calculated from the fluctuations of the winding number ${ }^{16} W$ for a system of size $L^{3}$ and is shown as a function of temperature in Fig. 1b. A finite-size scaling analysis of $\rho_{\mathrm{s}}$ gives the transition temperature $T_{\mathrm{c}}$, shown in Fig. 1a. It is also interesting to compare the superfluid density $\rho_{\mathrm{s}}$ with the condensate density $n_{0}$. In the superfluid phase, the single-particle density matrix $\left\langle b_{i}^{+} b_{j}\right\rangle$ approaches a constant equal to $n_{0}$ at large relative separation. The dependence of both $\rho_{\mathrm{s}}$ and $n_{0}$ on $t / U$ is shown in Fig. 1c. It is instructive to compare the behaviour of cold atoms in optical lattices with that of $\mathrm{He}^{4}$, in which the superfluid density $\rho_{\text {s }}$ approaches the total density at low temperatures, whereas the condensate density $n_{0}$ is only 0.1 . In optical lattices, both $\rho_{\mathrm{s}}$ and $n_{0}$ can be driven to zero at the quantum critical point by tuning the interactions.

For cold atoms in optical lattices, $\rho_{\mathrm{s}}$ has not been measured so far. We therefore focus on signatures of superfluidity in the momentum distribution. Turning to our calculations, we obtain the momentum distribution from equation (3) and input it in equation (2) to obtain the density distribution in the interference pattern at different temperatures and $t / U$. Figure 2 shows the evolution of the interference pattern for two lattice depths that correspond to the superfluid phase and close to the quantum critical point. Starting from a density profile that is a smooth gaussian at very high temperatures, a bimodal structure appears in the interference pattern, as $T$ decreases. Sharp peaks emerge above a broad background even when $T$ is still above $T_{\mathrm{c}}$ and the superfluid density is zero. The width of these peaks is narrower than the reciprocal lattice vector in a large temperature region above $T_{c}$. For example, in the right panel of Fig. $2 \mathrm{~b}$ the full-width at half-maximum at $t / U=0.25$ is already $1 / 8$ of the reciprocal lattice spacing at $T / T_{\mathrm{c}}=1.42$. Especially near the transition point $(t / U)_{c}$, sharp peaks can occur at temperatures much higher than $T_{\mathrm{c}}$.

It was pointed out by Diener et al. ${ }^{3}$ that for an ideal normal bosonic gas in an optical lattice, the interference pattern can have sharp features above $T_{\mathrm{c}}$. What is unexpected from the quantum Monte Carlo results is that even in a strongly interacting system close to the quantum critical point where interactions tend to broaden the peaks, it is nevertheless possible to see sharp peaks above $T_{\mathrm{c}}$. Starting in the normal state for a weakly interacting system, as $t / U$ is reduced at a fixed temperature $T_{0}$, and interactions between bosons dominate, the peaks in the interference pattern get broadened. At the same time, interactions have another effect of strongly suppressing $T_{\mathrm{c}}$ and in fact driving it to zero at the quantum critical point. So, if we now reduce $T$ below $T_{0}$, still remaining above $T_{c}$, the peaks are seen to sharpen and at the quantum critical point, as we explain below, the momentum distribution at small momentum diverges at $T=0$. Thus, it is clear that a bimodal distribution in the interference pattern that shows sharp peaks is not a signature for superfluidity, and using it as a criterion for superfluidity can lead to serious errors.

If we next consider the interference pattern in the left panel of Fig. 2b,d for $T<T_{c}$, we see the emergence of a $\delta$-function singularity over and above the regular sharp peaks that were already seen above $T_{\mathrm{c}}$. This singular peak breaks off from the already bimodal distribution above $T_{\mathrm{c}}$. Its height grows with the number of particles in the system and its width scales as $\approx 1 / L$, limited only by the system size $L$. It signals the emergence of the condensate and is the true and unequivocal signature of superfluidity. In the experiment, the presence of a confining harmonic trap makes the 
a

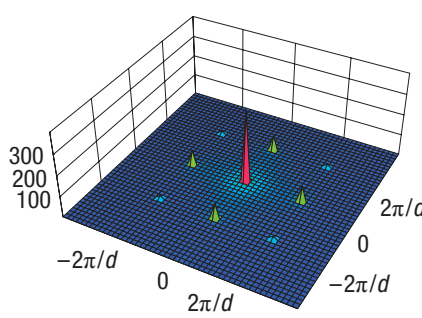

b

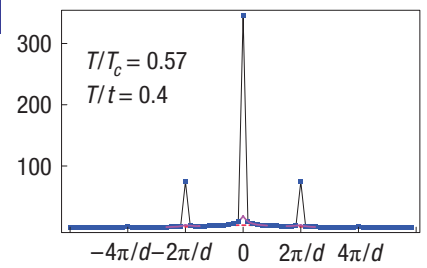

G

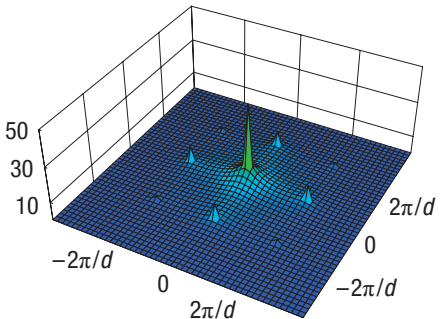

d

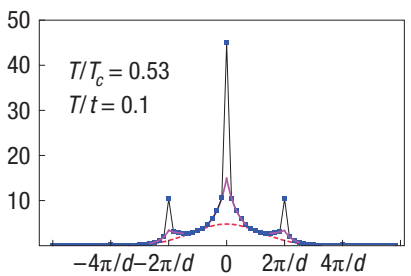

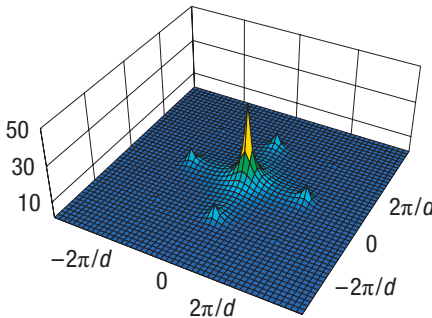
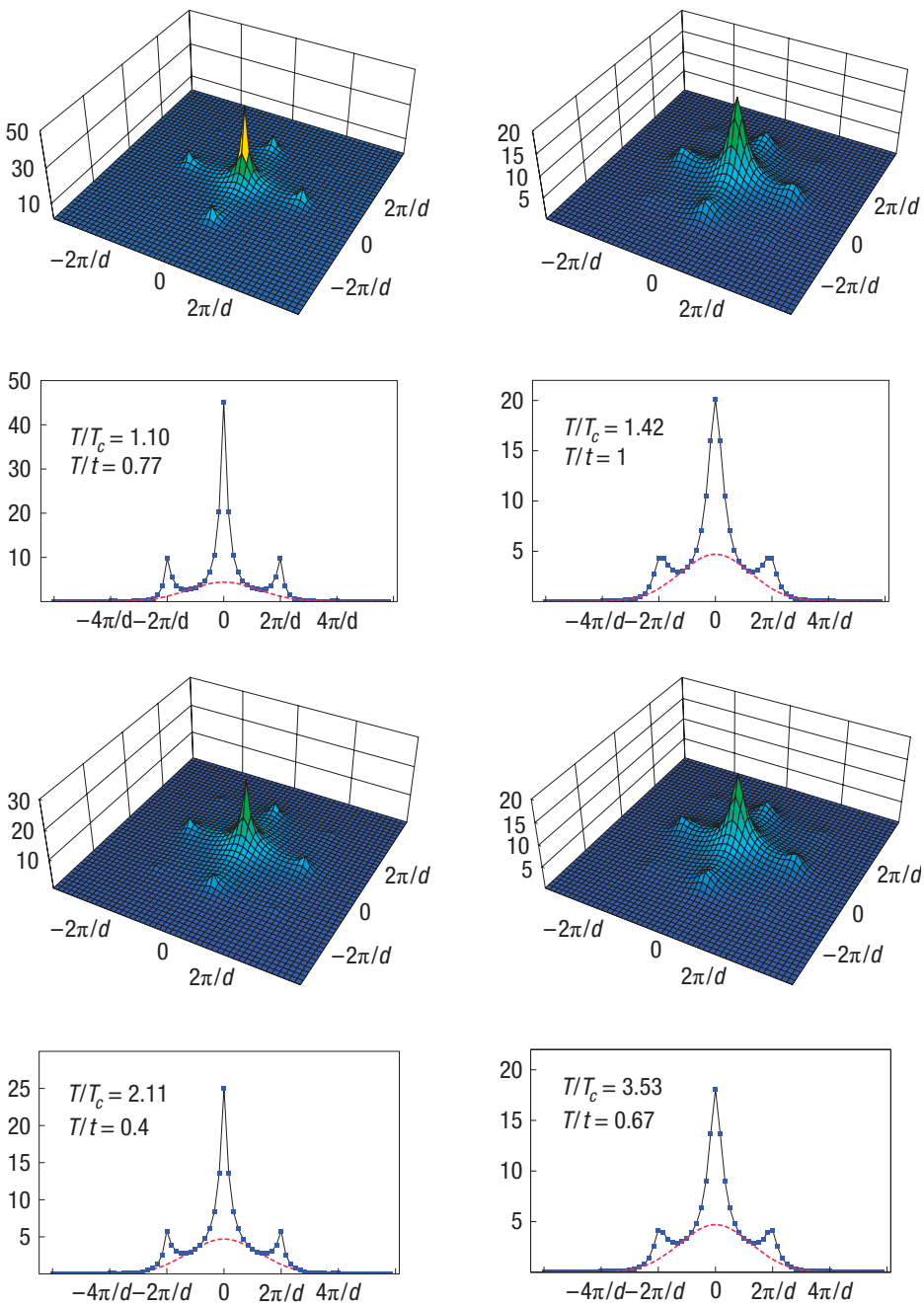

Figure 2 Interference pattern. a-d, The column-integrated momentum distribution $N_{\perp}\left(k_{x}, k_{y}\right)(\mathbf{a}, \mathbf{c})$ and the cut $N_{\perp}\left(k_{x}, k_{y}=0\right)(\mathbf{b}, \mathbf{d})$. The parameters in $\mathbf{a}$ and $\mathbf{b}$ are $t / U=0.25, V_{0}=11.5 E_{\mathrm{R}}$ and $T_{\mathrm{c}} / t=0.7$; in $\mathbf{c}$ and $\mathbf{d}, t / U=0.195, V_{0}=12.4 E_{\mathrm{R}}$ and $T_{\mathrm{c}} / t=0.19$. The clearly resolved sharp peaks, on top of the broad background, represented by the red dashed curve, in the central and right panels of $\mathbf{b}$ and $\mathbf{d}$ constitute a bimodal structure in the interference pattern above $T_{\mathrm{c}}$. In the left panel of $\mathbf{b}$ and $\mathbf{d}$, an extra peak arises, shown as a singular feature and leads to a trimodal structure below $T_{\mathrm{c}}$. Note that this $\delta$-function singular feature rises above the already sharp feature (shown in solid purple) that was present even above $T_{\mathrm{c}}$. The superfluid density $\rho_{\mathrm{s}}=0.44$ and 0.04 corresponding to the left column of $\mathbf{b}$ and $\mathbf{d}$, respectively. For all other columns, $\rho_{\mathrm{s}}=0$. The lattice size is $N_{\mathrm{L}}=12^{3}$.
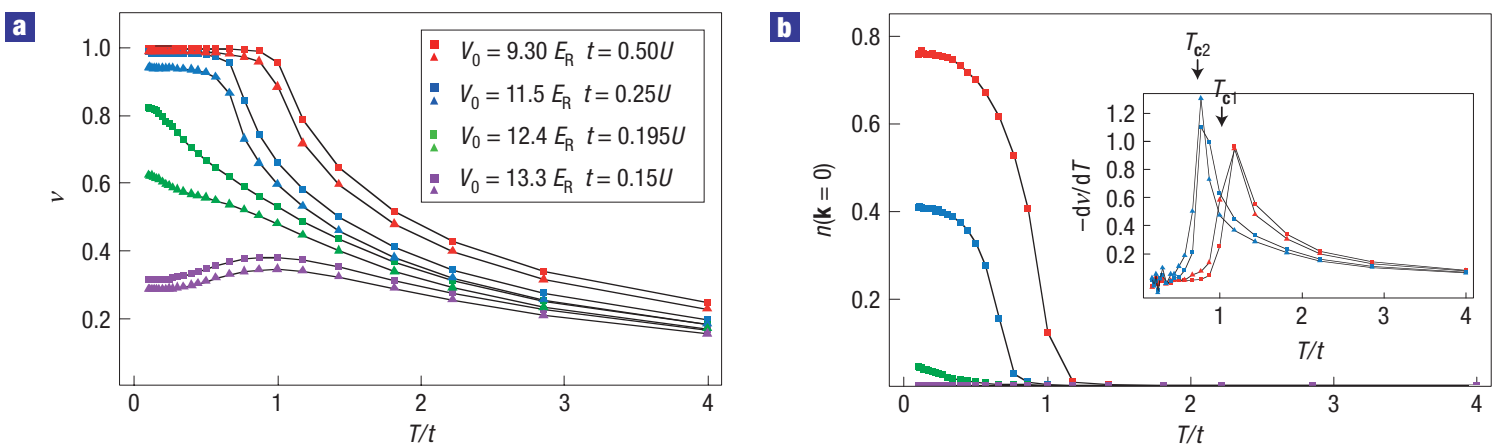

Figure 3 Visibility. a, $v$ defined in equation (4) for a system of size $N_{\mathrm{L}}=12^{3}$. For each parameter $V_{0} / E_{R}$, the two curves with the same colour of symbols correspond to averaging over different bin sizes $\delta k$. The squares correspond to $\delta k=0$ and the triangles to $\delta k=\pi / 6$. In the superfluid phase, the visibility becomes almost unity below $T_{\mathrm{c}}$ and is not much affected by small $\delta k . \mathbf{b}$, The corresponding zero momentum population $n(\mathbf{k}=0)$. The inset shows that $T_{\mathrm{c}}$ can be estimated roughly from the peak in the $T$-derivative of the visibility. $T_{\mathrm{c} 1}=1 \mathrm{t}$ and $T_{\mathrm{c} 2}=0.7 t$ are the critical temperatures from Fig. 1 for $V_{0}=9.30 E_{\mathrm{R}}$ and $V_{0}=11.5 E_{\mathrm{R}}$, respectively. 

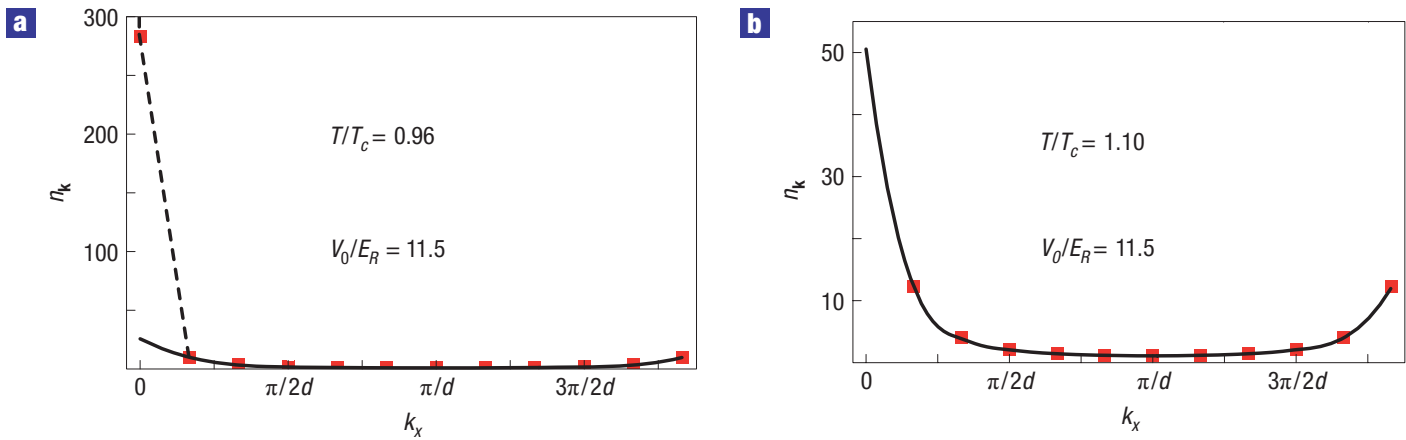

Figure 4 Crystal momentum distribution. $n\left(k_{x}, 0,0\right)$ defined in equation (5) for a system of size $N_{\mathrm{L}}=12^{3}$ at $t / U=0.25$. a, $T / T_{\mathrm{c}}=0.96$ showing a distinct condensate $N_{0}$ that is singular and breaks off from a smooth background $n_{\text {reg }} . \mathbf{b}, T / T_{\mathrm{c}}=1.1$ showing that the singular contribution $N_{0}$ is absent. Note that the regular contribution $n_{\text {reg }}(\mathbf{k} \rightarrow 0)$ can become large close to the transition.

a

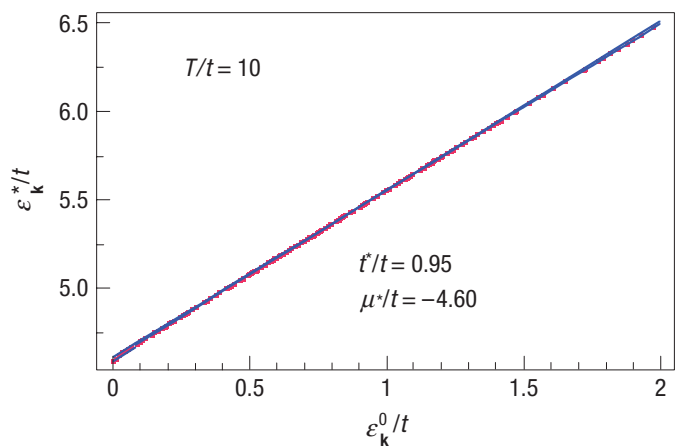

$\mathbf{6}$

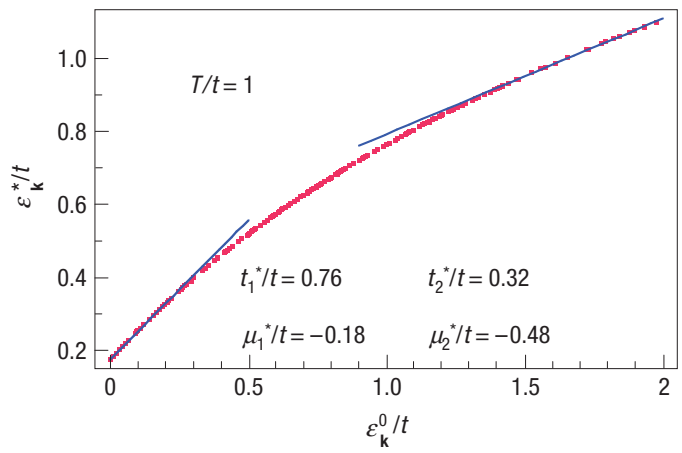

b

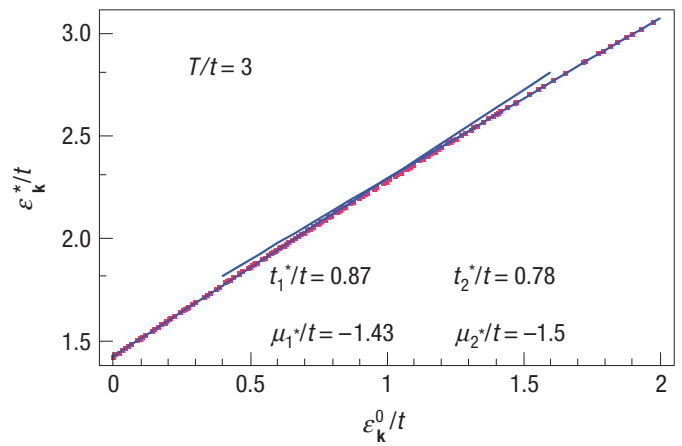

d

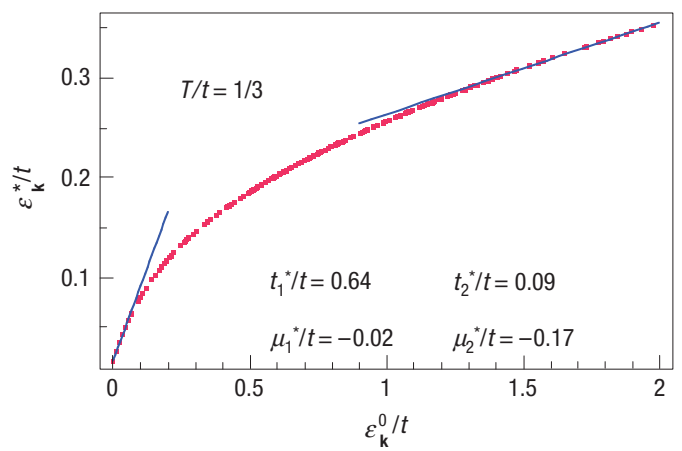

Figure 5 Momentum distribution function at the quantum critical point. $n(\mathbf{k})$ for a $N_{\mathrm{L}}=24^{3}$ system is plotted by extracting the effective dispersion $\varepsilon_{\mathbf{k}}^{*} \equiv T \log (1+1 / n(\mathbf{k}))$ in analogy with the free boson $n_{\text {rree }}(\mathbf{k}) \mathbf{a}$, At high $T=10 t$, the almost linear fit indicates that the bosons are well described as free particles with weakly renormalized parameters. b-d, As $T$ is lowered to $T=3 t(\mathbf{b}), T=t(\mathbf{c})$ and $T=t / 3(\mathbf{d})$, there are distinct deviations from linearity that increase with decreasing $T$. Separate linear fits to the low- $\left(t_{1}^{*}\right.$ and $\left.\mu_{1}^{*}\right)$ and high- $\left(t_{2}^{*}\right.$ and $\left.\mu_{2}^{*}\right)$ energy parts of the spectrum show strong renormalizations of the effective bandwidth and chemical potential.

system inhomogeneous, and leads to the coexistence of multiple phases. In such a situation, the interference pattern should be examined more carefully to detect the superfluid in the system, as the width of the peak scales as the inverse of the linear size of the superfluid region.

To quantitatively analyse the interference pattern, the visibility is measured in many experiments ${ }^{7,8,17}$ defined by,

$$
v=\frac{N_{\mathrm{A}}-N_{\mathrm{B}}}{N_{\mathrm{A}}+N_{\mathrm{B}}}
$$

where $N_{\mathrm{A}}=N_{\perp}(G \hat{x}), N_{\mathrm{B}}=N_{\perp}(G \hat{n}), G=2 \pi / d$ and $G \hat{x}$ is a reciprocal lattice vector; $G \hat{n}$ is $G \hat{x}$ rotated by $45^{\circ}$ around the $\hat{z}$ axis. The robust feature about the visibility is that in the superfluid phase, $v=1$ dominated by the macroscopic number of atoms in the condensate $N_{\mathrm{A}} \approx O(N)$. This result does not change if we include a finite bin size and average $N_{\perp}$ over a small area for both $\mathrm{A}$ and $\mathrm{B}$. Certainly if the simulation size is small and the condensate does not contain a macroscopic number of particles, then changing the bin size can significantly affect the visibility and obscure the superfluid phase $^{18}$. In our simulations on $12^{3}$ size lattices, even with a bin 
size $\delta k=1 / 12$ of the reciprocal lattice vector, the visibility remains robust at unity for $V_{0}=9.3 E_{\mathrm{R}}$ and 0.94 for $V_{0}=11.5 E_{\mathrm{R}}$, which is already very close to the quantum critical point $V_{\mathrm{c}}=12.5 E_{\mathrm{R}}$. Here $E_{\mathrm{R}}=h^{2} / 8 m d^{2}$ is the recoil energy in terms of the optical lattice spacing $d$ and mass of atoms $m$. We expect the visibility in the experiments with $50^{3}-70^{3}$ lattice sites to be very robust at unity below $T_{\mathrm{c}}$. Another salient feature of the visibility is that it is almost $T$ independent below $T_{\mathrm{c}}$, because $n(\mathbf{k}=0)$ decreases slowly below $T_{c}$, as shown in Fig. 3b. On the other hand, it decreases rapidly near $T_{c}$, enabling us to roughly estimate $T_{c}$ from the peak in $\mathrm{d} v / \mathrm{d} T$. At the quantum critical point, $v$ can also be fairly high owing to the large population as $\mathbf{k} \rightarrow 0$ even though there is no singularity in $n(\mathbf{k})$.

In Fig. 2, both quantum and thermal fluctuations result in uncondensed bosons with a contribution that is convoluted with the Wannier function to produce the broad gaussian background. We directly analyse the crystal momentum distribution $n(\mathbf{k})$, without any obfuscation from the Wannier function, in the first Brillouin zone before expansion ${ }^{19}$. Figure $4 \mathrm{a}, \mathrm{b}$ shows the crystal momentum distribution just above and below $T_{\mathrm{c}}$. In the superfluid phase

$$
n(\mathbf{k})=N_{0} \delta(\mathbf{k}=0)+n_{\text {reg }}(\mathbf{k}),
$$

where $N_{0}$ is the number of Bose condensed atoms at $\mathbf{k}=0$, whereas $n_{\text {reg }}(\mathbf{k})$ is the regular uncondensed bosons. When the temperature goes above $T_{c}$, the $\delta$-function singular part $N_{0}$ disappears, which indicates the vanishing of superfluid order (Fig. $4 \mathrm{~b}$ ). However, in a strongly correlated superfluid close to the quantum critical point, $n_{\text {reg }}(\mathbf{k} \rightarrow 0)$ can become large, resulting in sharp peaks in the corresponding interference pattern. The fact that $n(\mathbf{k})$ can become sharply peaked but not singular at temperatures above $T_{\mathrm{c}}$ was also found in neutron scattering experiments ${ }^{20}$ on $\mathrm{He}^{4}$.

In analogy with the non-interacting boson distribution, Fig. 5 shows $\varepsilon_{\mathbf{k}}^{*} \equiv T \log (1+1 / n(\mathbf{k}))$ as a function of $\varepsilon_{\mathbf{k}}^{0}$ for various $T$ at $(t / U)_{c}$, where $\varepsilon_{\mathrm{k}}^{0}=(t / 3) \sum_{\mu=x, y, z}\left(1-\cos \left(k_{\mu} d\right)\right)$ is the bare single-particle energy. At high $T=10 t$, we obtain a linear fit, which implies that the boson distribution is well described by

$$
n_{\text {free }}(\mathbf{k})=\frac{1}{\mathrm{e}^{\beta\left(\varepsilon_{\mathbf{k}}^{*}-\mu^{*}\right)}-1}
$$

as weakly interacting particles. Here, $\varepsilon_{\mathbf{k}}^{*}=\left(t^{*} / 3\right) \sum_{\mu=x, y, z}$ $\left(1-\cos \left(k_{\mu} d\right)\right)$ is the renormalized dispersion arising from a renormalized bandwidth $t^{*}$ and a renormalized chemical potential $\mu^{*}$. At $T=3 t$, deviations are seen from linear behaviour, which grow with decreasing $T$. The slope of the curve changes smoothly enabling linear fits to the low- (with parameters $t_{1}^{*}$ and $\mu_{1}^{*}$ ) and high- (with parameters $t_{2}^{*}$ and $\mu_{2}^{*}$ ) energy regimes that reveal strong renormalizations of the parameters. The significance of this finding is that in the low-energy regime $n(\mathbf{k})$ from equation (6) can be described by $n(\mathbf{k})=\left(T /\left|\mu_{1}^{*}\right|\right) /\left(1+k^{2} \xi^{2}\right)$ at the quantum critical point where $\left|\mu_{1}^{*}\right|$ is found to be much less than $T$ and $t_{1}^{*}$ and the healing length is $\xi(T) / d=\sqrt{t_{1}^{*} /\left(6\left|\mu_{1}^{*}\right|\right)}$. At the quantum critical point as $T \rightarrow 0$, we find that $\mu_{1}^{*} / T \rightarrow 0$ but $t_{1}^{*}$ is finite resulting in a correlation length $\xi(T)$ that becomes larger with decreasing $T$ and finally diverges.

The precise form of the dispersion and spectral function of bosons in optical lattices are of fundamental interest. We hope our computational and theoretical analysis will inspire further investigations of strongly interacting systems, especially in the quantum critical region ${ }^{21}$.

Received 9 February 2008; accepted 6 May 2008; published 30 May 2008.

References

1. Bloch, I. Ultracold Quantum Gases in optical lattices. Nature Phys. 1, 23-30 (2005).

2. Bloch, I., Dalibard, J. \& Zwerger, W. Many-body physics with ultracold gases. Rev. Mod. Phys. (in the press); preprint at $<$ http://arxiv.org/abs/0704.3011> (2007).

3. Diener, R. B., Zhou, Q., Zhai, H. \& Ho, T. L. Criterion for bosonic superfluidity in an optical lattice. Phys. Rev. Lett. 98, 180404 (2007).

4. Xu, K. et al. Observation of strong quantum depletion in a gaseous Bose-Einstein condensate. Phys. Rev. Lett. 96, 180405 (2006).

5. Greiner, M., Mandel, O., Esslinger, T., Hänsch, T. W. \& Bloch, I. Quantum phase transition from a superfluid to a Mott insulator in a gas of ultracold atoms. Nature 415, 39-44 (2002).

6. Chin, J. K. et al. Evidence for superfluidity of ultracold fermions in an optical lattice. Nature $\mathbf{4 4 3}$, 961-964 (2006).

7. Günter, K., Stöferle, T., Moritz, H., Köhl, M. \& Esslinger, T. Bose-Fermi mixtures in a three-dimensional optical lattice. Phys. Rev. Lett. 96, 180402 (2006).

8. Ospelkaus, S. et al. Localization of bosonic atoms by fermionic impurities in a three-dimensional optical lattice. Phys. Rev. Lett. 96, 180403 (2006).

9. Fisher, M. P. A., Weichman, P. B., Grinstein, G. \& Fisher, D. S. Boson localization and the superfluid-insulator transition. Phys. Rev. B. 40, 546-570 (1989).

10. Jaksch, D., Bruder, C., Cirac, J. I., Gardiner, C. W. \& Zoller, P. Cold bosonic atoms in optical lattices. Phys. Rev. Lett. 81, 3108-3111 (1998).

11. Pedri, P. et al. Expansion of a coherent array of Bose-Einstein condensates. Phys. Rev. Lett. 87, 220401 (2001).

12. Gerbier, F. et al. Interference pattern and visibility of a Mott insulator. Phys. Rev. A. 72, 053606 (2005).

13. Capogrosso-Sansone, B., Prokof'ev, N. V. \& Svistunov, B. V. Phase diagram and thermodynamics of the three-dimensional Bose-Hubbard model. Phys. Rev. B. 75, 134302 (1998).

14. Syljuasen, O. F. \& Sandvik, A. W. Quantum Monte Carlo with directed loops. Phys. Rev. E. 66, 046701 (2002).

15. Kato, Y., Suzuki, T. \& Kawashima, N. Modification of directed-loop algorithm for continuous space simulation of bosonic systems. Phys. Rev. E. 75, 066703 (2007).

16. Pollock, E. L. \& Ceperley, D. M. Path-integral computation of superfluid densities. Phys. Rev. B. 36, 8343-8352 (1987).

17. Gerbier, F. et al. Phase coherence of an atomic Mott insulator. Phys. Rev. Lett. 95, 050404 (2005).

18. Pollet, L., Kollath, C., Houcke, K. V. \& Troyer, M. Temperature changes when adiabatically ramping up an optical lattice. New. J. Phys. (in the press); preprint at $<$ http://arxiv.org/abs/0801.1887> (2008).

19. Spielman, I. B., Phillips, W. D. \& Porto, J. V. Mott-insulator transition in a two-dimensional atomic Bose gas. Phys. Rev. Lett. 98, 080404 (2007).

20. Sokol, P. E. in Bose-Einstein Condensation (eds Griffin, A., Snoke, D. W. \& Stringari, S.) (Cambridge Univ. Press, Cambridge, 1995).

21. Sachdev, S. Quantum Phase Transitions (Cambridge Univ. Press, Cambridge, 1999).

\section{Acknowledgements}

We would like to especially thank T.-L. Ho and I. Bloch for many stimulating discussions. The simulation was carried out at the Supercomputer Center, Institute for Solid State Physics, University of Tokyo. Y.K. and N.K. are financially supported by KAKENHI 19340109 and 19052004, and by the Next Generation Supercomputing Project, Nanoscience Program, MEXT, Japan. We also thank the Institute for Complex Adaptive Matter for partial financial support.

Author information

Reprints and permission information is available online at http://npg.nature.com/reprintsandpermissions. Correspondence and requests for materials should be addressed to N.T. 